\title{
A Patient with Tubo-Ovarian Abscess with Endometriosis after Transvaginal Oocyte Retrieval
}

\author{
Tsutomu Muramoto, Ryo Koike \\ Department of Obstetrics and Gynecology, Showa University Fujigaoka Hospital, \\ Yokohama, Japan \\ Email: t_muramo@yahoo.co.jp
}

How to cite this paper: Muramoto, T. and Koike, R. (2020) A Patient with Tubo-Ovarian Abscess with Endometriosis after Transvaginal Oocyte Retrieval. Open Journal of Obstetrics and Gynecology, 10, 445-451. https://doi.org/10.4236/ojog.2020.1040040

Received: March 14, 2020

Accepted: April 5, 2020

Published: April 8, 2020

Copyright (C) 2020 by author(s) and Scientific Research Publishing Inc. This work is licensed under the Creative Commons Attribution International License (CC BY 4.0).

http://creativecommons.org/licenses/by/4.0/ (c) (i) Open Access

\begin{abstract}
Ultrasound-guided oocyte retrieval is a standard procedure for ovum pick up (OPU) during in vitro fertilization. Possible complications include tubo-ovarian abscesses (TOAs) in the fallopian tubes, and endometriosis may be associated with increasing the severity of pelvic infection. A 35-year-old woman who had been treated for infertility and endometriosis presented with abdominal pain that appeared several days after OPU. There were no significant changes in the size of both ovaries and fallopian tubes. A week later, transvaginal echography revealed right oviduct enlargement. Because conservative treatment with antibiotics before this revelation had not been successful, laparoscopy was performed for abscess drainage and/or excision of the adnexa. Intraoperative findings were an abscess in the right adnexa involving the ovary and fallopian tube. Preservation of the right fallopian tube was difficult due to the risk of prolonged infection, and a right appendectomy was performed. The diagnosis was a right TOA. Severe cases of pelvic infection after OPU may cause infertility. The presence of endometriosis may aggravate the infection, but the relationship between the extent of the endometriosis and the severity of the infection is not known. Even if the endometriosis is mild, as it was in this patient, surgical treatment should be considered if a severe infection occurs after OPU and conservative treatment is not effective.
\end{abstract}

\section{Keywords}

Tubo-Ovarian Abscess, Transvaginal Oocyte Retrieval, Endometriosis

\section{Introduction}

Transvaginal echo-guided egg harvesting is known to cause complications such 
as organ damage, hemorrhaging, and pelvic infections. The frequency of complications is low. Post-puncture infections occur following less than $1 \%$ of procedures, but severe infections may result in pelvic or tubal abscesses. If conservative treatment by antimicrobial agents is not effective, then surgical treatments such as abscess drainage and/or source clearance of the infection and extraction is necessary. Tubal abscesses may be associated with pelvic inflammatory diseases or endometriosis. The presence of endometriosis may aggravate infections, but the relationship between the extent of the endometriosis and the severity of infection is not known. Even small endometriotic cysts, as was seen in this study patient, could eventually become fallopian tube abscesses due to infection after transvaginal ultrasound-guided ovum pick up (OPU); and this patient was treated by laparoscopic surgery.

\section{Case Presentation}

The patient was a 35-year-old G0P0 woman with a 30 - day menstrual cycle. Her history included past treatment at another hospital for infertility. Endometriotic cysts that had been detected were noted but not treated due to their small, 10 $20 \mathrm{~mm}$ size. Eggs had been collected two months prior, and were followed by infection prophylaxis with a one-time administration of a $1 \mathrm{~g}$ fosfomycin drip and the oral administration of levofloxacin $500 \mathrm{mg} /$ day for seven days. Bilateral intimal cysts that were, $20 \mathrm{~mm}$ on the left and $10 \mathrm{~mm}$ on the right side were seen through ultrasound at the time of egg collection. No remarkable changes since the last evaluation were noted; no cysts were punctured at the time of egg collection. The patient experienced mild low abdominal pain with a body temperature of $37^{\circ} \mathrm{C}$ for several days before the scheduled embryo transfer. There was no evidence of fallopian tube and ovarian enlargement as confirmed on either side through ultrasonography. The embryo transfer scheduled for this cycle was postponed. A week later, the enlargement of the right fallopian tube (long axis $77 \mathrm{~mm}$ ) was seen on a transvaginal echogram together with a white blood cell count (WBC) OF $8000 / \mu \mathrm{L}$ and a C-reactive protein (CRP) of $11.88 \mathrm{mg} / \mathrm{dL}$, thus indicative of inflammation. Levofloxacin $500 \mathrm{mg} /$ day resulted in the improvement of symptoms, fever, and lower abdominal pain after two days. After four days, echograms showed oviduct enlargement (long diameter $85 \mathrm{~mm}$ ). The WBC was $9000 / \mu \mathrm{L}$. The patient was referred to our department for the treatment of a right tubo-ovarian abscess. Her surgical history included a laparoscopic cystectomy to treat endometriotic cysts at another hospital at age 32 (details unknown).

Enhanced abdominal computed tomography (CT) revealed a multilocular cyst that appeared to contain pus on the dorsal aspect of the right appendage of the uterus. The abscess involved in the right whole tube and ovary (Figure 1).

Because conservative treatment with antibiotics before this revelation had not been successful, laparoscopy was performed for abscess drainage and or excision of the adnexa. Intraoperative findings were an abscess in the right adnexa involving the ovary and fallopian tube. The sigmoid colon was adherent to the bilateral 


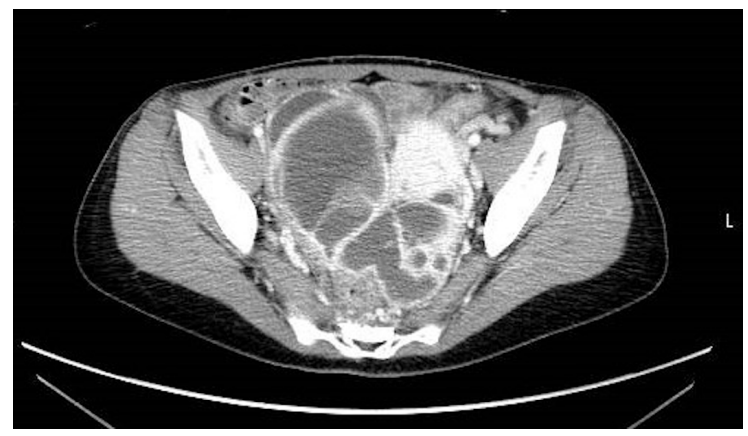

Figure 1. Enhanced abdominal computed tomography.

sacral uterine ligaments on the dorsal aspect of the uterus and the left ovary. Preservation of the right fallopian tube was difficult and involved a risk of prolonged infection. The adhesions between the abscess and the posterior surface of the uterus and the sigmoid colon were removed, and right appendectomy was performed. Adhesions of the left appendage and ovary were dissected, and the surgery was completed by placing a drain in the pouch of Douglas. The postoperative course was uneventful with improvement of lower abdominal pain on day 1. Tazobactam/Piperacillin $13.5 \mathrm{~g} /$ day for three days were administered as antimicrobial agents after the surgery. Pelvic drainage decreased over time, and the drain was removed on day 3 . The patient was discharged on day 8 following laboratory evidence of a decrease in the inflammatory reaction (WBC and CRP). Escherichia coli was identified in cultures of the abscess contents. The recurrence of pelvic abscess has not occurred in the 12 months following surgery.

\section{Discussion}

Ultrasound-guided egg collection is a standard in vitro fertilization procedure in assisted reproductive medicine [1]. Although the procedure is relatively easy and safe, it is associated with complications such as damage to pelvic organs, hemorrhaging, and pelvic infections [2]. Organ damage is extremely rare; puncture-associated bleeding occurs in a small percentage of procedures, and the incidence of pelvic infection after OPU is less than 1\% [3] [4] [5]. The route of infection may start in the vagina or uterus, and antibiotic prophylaxis may be effective, but rare, severe pelvic abscesses may still form [6]. If conservative treatments with antibiotics are not successful, surgical CT- or echo-guided transvaginal abscess drainage or laparoscopic excision is performed [7]. If the ovaries and fallopian tubes are involved, and antibiotics and abscess drainage are insufficient, reoperation and appendectomy may be necessary. Conservative treatments tend not to be effective in patients with tubo-ovarian abscesses larger than $5 \mathrm{~cm}$ in diameter or with severe inflammation on blood evaluation [7]. Drainage or surgical removal is then necessary [8]. Approximately $25 \%$ - $40 \%$ of infertile women have endometriosis, which may be associated with an increased severity in infections and the formation of abscesses [9] [10]. Cyst walls are more fragile and more easily infected than normal ovarian epithelium. The content of endo- 
metriotic cysts includes blood, and the environment within the cyst is anaerobic [6] [11]. The cyst environment thus favors bacterial growth, and the low penetration of antibiotics into cysts increases the likelihood that surgery will ultimately be required. Endometriotic cysts are susceptible to infection, but there is no known correlation between the size of cysts and the incidence and severity of pelvic infections [11]. However, the characteristics of endometriotic cysts, the fragility of the cyst wall, and an environment that supports bacterial growth are consistent with such a relationship [8]. Even if the diameter of endometriotic cysts is small as in this case, an infection may become severe and lead to TOA after OPU. A larger tumor diameter at the time of surgery would be associated with more severe infection and a longer postoperative hospital stay, however, endometriotic cysts may suddenly increase after egg collection as they were in this case [6] [7]. We must cautiously look out for postoperative infections after OPU regardless of the diameter of the endometriotic cyst. Gardella vaginalis, Enterococcus sp., Streptococcus sp., E. coli, and Candida Albicans have been reported in TOAs [12] [13] [14]. Pathogens are often not isolated in cultures, but E. coli was isolated in this patient, and the possibility of an ascending route of infection was considered. An association between endometriosis and the severity of infection after egg collection has been reported, but the extent of the endometriosis' influence is not clear [6]. Small endometriotic cysts could lead to the aggravation of subsequent infections; however, surgery for endometriotic cysts might cause postoperative ovarian malfunctions [15]. We do not recommend surgery for the removal of small endometrial cysts, but laparoscopic evaluations to determine the extent of endometriosis and its potential influence on fertility are recommended [16]. Surgery may be indicated for large endometrial cysts, but the risk of recurrence and loss of the ovarian reservation must be considered. The time of egg collection following surgery is affected because a relapse of intimal disease is possible, and the likelihood of pregnancy may decrease due to increases in patient age. Since the incidence of infection in the interval between surgery and egg collection is not known, patient age, infertility, and intima status must be considered when performing egg collection after performing surgeries to treat endometriosis. Treatments need to be adjusted on a case-by-case basis depending on the severity of the patient's condition. In addition, in patients with a history of surgery, reoperation may reduce the egg reserve. The risk of infection is thought to be low in patients with small endometriotic cysts, and treatment of the intima prior to egg collection may not be necessary. Cyst infection may recur after prior surgeries, but antimicrobial treatments and drainage are possible at that time. A previous report describes a case in which pregnancy was achieved following embryo transfer, and endometriotic cyst removal was performed afterward [6]. In that patient, an endometriotic cyst was punctured during egg collection and an ovarian abscess developed. It is not clear whether cyst puncture during egg collection increases the risk of formation of a TOA. In this patient, the endometriotic cysts were small and were not punctured. Conserva- 
tive treatments of TOAs with antibiotics, minimally invasive treatment such as echo-guided transvaginal abscess drainage, and laparoscopic or open surgery for severe cases caused by endometriosis are available. Organ removal is also an option. Antibiotic prophylaxis may be administered at the time of egg collection. Broad-spectrum antibiotics are recommended because both aerobic Gram-negative bacilli and Gram-positive cocci have been isolated from infections following egg collection. However, if the infection becomes severe and a TOA occurs, only about $70 \%$ of the infections are resolved with conservative treatment, and surgery is often necessary. Transvaginal abscess drainage may improve the response to antibiotics, but there are reports that conservative treatments were not effective in cases that had previously undergone intravaginal drainage and ultimately required open cystectomies [17]. Infertility may be associated with prolonged adhesion and pelvic inflammation. If conservative treatments of severe infection are not effective, prompt surgery is recommended. In this patient, antimicrobial prophylaxis and the conservative treatment for an infection following egg collection did not prevent TOA formation. Surgery was required and was successful. Caution is necessary because even small endometrial cysts can cause TOAs. If an abscess occurs, transvaginal echo-guided abscess drainage may be effective, but if there is intimal disease or if inflammation is prolonged, there is a high possibility of adhesion in the surrounding organs. Laparoscopic drainage of abscesses should be considered in such TOA patients. Laparoscopic surgery is less invasive than laparotomies, and features less blood loss, shorter hospitalization, less postoperative fever, and fewer intraoperative complications [18]. However, in patients with intestinal tract adhesions or generalized peritonitis, switching to a laparotomy is recommended. This patient had a right ovary abscess with a marked dilation of the right fallopian tube. The diagnosis was a right TOA. A laparoscopic right appendectomy was performed because the inflammation was severe, and infection control with a cystectomy alone was thought to be insufficient in avoiding relapse. The pelvic adhesions were extensive, but surgery was successful without causing intestinal damage. As with intimal surgery, surgery for fallopian tube abscesses need to preserve normal ovary tissue to avoid the postoperative loss in the ovarian reservation. Precautions include removal of the source of infection to avoid a subsequent need for a hysterectomy.

\section{Conclusion}

A patient with endometriotic cysts who developed a fallopian tube abscess after echo-guided egg collection was treated with laparoscopic surgery. Pelvic infections are a rare complication of egg collection, and severe cases require surgical treatment. Endometriosis may aggravate infections, but the relation between the extent of intima involvement and the severity of infection is not known. Even small endometriotic cysts as found in this case can eventually become involved with fallopian tube abscesses. Prompt surgical treatment should be considered if conservative treatments are not effective in treating severe infections. 


\section{Contributions}

These authors contributed equally to this work.

Muramoto and Koike made diagnose of this patient and performing operation together.

Koike gave Muramoto advice in preparing the dissertation considerations.

\section{Consent}

The patient had given her own informed consent for the case report to be published.

\section{Conflicts of Interest}

The authors declare no conflicts of interest regarding the publication of this paper.

\section{References}

[1] Wikland, M., Enk, L. and Hamberger, L. (1985) Transvesical and Transvaginal Approaches for the Aspiration of Follicles by Use of Ultrasound. Annals of the New York Academy of Sciences, 442, 182-194.

https://doi.org/10.1111/j.1749-6632.1985.tb37519.x

[2] Dicker, D., Ashkenazi, J., Feldberg, D., et al. (1993) Severe Abdominal Complications after Transvaginal Ultrasonographically Guided Retrieval of Oocytes for in Vitro Fertilization and Embryo Transfer. Fertility and Sterility, 59, 1313-1315. https://doi.org/10.1016/S0015-0282(16)55997-4

[3] Bennett, S.J., Waterstone, J.J., Cheng, W.C., et al. (1993) Complications of Transvaginal Ultrasound-Directed Follicle Aspiration: A Review of 2670 Consecutive Procedures. Journal of Assisted Reproduction and Genetics, 10, 72-77. https://doi.org/10.1007/BF01204444

[4] Bodri, D., Guillén, J.J., Polo, A., et al. (2008) Complications Related to Ovarian Stimulation and Oocyte Retrieval in 4052 Oocyte Donor Cycles. Eproductive BioMedicine Online, 17, 237-243. https://doi.org/10.1016/S1472-6483(10)60200-3

[5] Ludwig, A.K., Glawatz, M., Griesinger, G., et al. (2006) Perioperative and Post-Operative Complications of Transvaginal Ultrasound-Guided Oocyte Retrieval: Prospective Study of > 1000 Oocyte Retrievals. Human Reproduction, 21, 3235-3240. https://doi.org/10.1093/humrep/del278

[6] Moini, A., Riazi, K., Amid, V., et al. (2005) Endometriosis May Contribute to Oocyte Retrieval-Induced Pelvic Inflammatory Disease: Report of Eight Cases. Journal of Assisted Reproduction and Genetics, 22, 307-309.

https://doi.org/10.1007/s10815-005-6003-2

[7] Dewitt, J., Reining, A., Allsworth, J.E., et al. (2010) Tuboovarian Abscesses: Is Size Associated with Duration of Hospitalization \& Complications? Obstetrics and Gynecology International, 2010, Article ID: 847041. https://doi.org/10.1155/2010/847041

[8] Akkurt, M.Ö., Yalçın, S.E., Akkurt, İ., et al. (2015) The Evaluation of Risk Factors for Failed Response to Conservative Treatment in Tubo-Ovarian Abscesses. Journal of the Turkish-German Gynecological Association, 16, 226-230.

https://doi.org/10.5152/jtgga.2015.15123

[9] Hickman, T.N. (2002) Impact of Endometriosis on Implantation. Data from the 
Wilford Hall Medical Center IVFET Program. The Journal of Reproductive Medicine, 47, 801-808.

[10] Laganà, A.S., Vitale, S.G., Salmeri, F.M., et al. (2017) Unus pro omnibus, omnes pro uno: A Novel, Evidence-Based, Unifying Theory for the Pathogenesis of Endometriosis. Medical Hypotheses, 103, 10-20. https://doi.org/10.1016/j.mehy.2017.03.032

[11] Chen, M.J., Yang, J.H., Yang, Y.S., et al. (2004) Increased Occurrence of Tubo-Ovarian Abscesses in Women with Stage III and IV Endometriosis. Fertility and Sterility, 82, 498-499. https://doi.org/10.1016/j.fertnstert.2004.01.032

[12] Kavoussi, S.K., Pearlman, M.D., Burke, W.M., et al. (2006) Endometrioma Complicated by Tubo-Ovarian Abscess in a Woman with Bacterial Vaginosis. Infectious Diseases in Obstetrics and Gynecology, 2006, Article ID: 84140.

https://doi.org/10.1155/IDOG/2006/84140

[13] Sharpe, K., Karovitch, A.J., Claman, P., et al. (2006) Transvaginal Oocyte Retrieval for in Vitro Fertilization Complicated by Ovarian Abscess during Pregnancy. Fertility and Sterility, 86, 219.e11-3. https://doi.org/10.1016/j.fertnstert.2005.12.045

[14] To, V., Gurberg, J. and Krishnamurthy, S. (2015) Tubo-Ovarian Abscess Caused by Candida Albicans in an Obese Patient. Journal of Obstetrics and Gynaecology Canada, 37, 426-429. https://doi.org/10.1016/S1701-2163(15)30257-7

[15] Somigliana, E., Ragni, G., Benedetti, F., et al. (2003) Does Laparoscopic Excision of Endometriotic Ovarian Cysts Significantly Affect Ovarian Reserve? Insights from IVF Cycles. Human Reproduction, 18, 2450-2453.

https://doi.org/10.1093/humrep/deg432

[16] Hachisuga, T. and Kawarabayashi, T. (2002) Histopathological Analysis of Laparoscopically Treated Ovarian Endometriotic Cysts with Special Reference to Loss of Follicles. Human Reproduction, 17, 432-435. https://doi.org/10.1093/humrep/17.2.432

[17] Greenstein, Y., Shah, A.J., Vragovic, O., et al. (2013) Tuboovarian Abscess. Factors Associated with Operative Intervention after Failed Antibiotic Therapy. The Journal of Reproductive Medicine, 58, 101-106.

[18] Sciarra, J.J. (2006) Endoscopy in Gynecology: Past, Present, and Future. Journal of Minimally Invasive Gynecology, 13, 367-369.

https://doi.org/10.1016/j.jmig.2006.06.028 